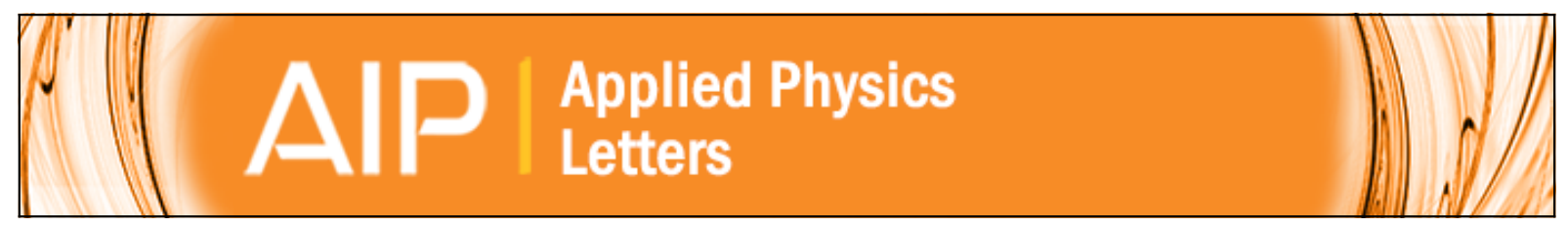

\title{
Many-body effects on excitons properties in GaN/AIGaN quantum wells
}

Giampiero Traetta, Roberto Cingolani, Aldo Di Carlo, Fabio Della Sala, and Paolo Lugli

Citation: Applied Physics Letters 76, 1042 (2000); doi: 10.1063/1.125932

View online: http://dx.doi.org/10.1063/1.125932

View Table of Contents: http://scitation.aip.org/content/aip/journal/apl/76/8?ver=pdfcov

Published by the AIP Publishing

\section{Articles you may be interested in}

Laser field and electric field effects on exciton states and optical properties in zinc-blende GaN/AIGaN quantum well

J. Appl. Phys. 113, 214310 (2013); 10.1063/1.4809749

Terahertz intersubband transition in GaN/AIGaN step quantum well

J. Appl. Phys. 113, 154505 (2013); 10.1063/1.4802496

Ultrafast differential transmission spectroscopy of excitonic transitions in InGaN/GaN multiple quantum wells J. Appl. Phys. 93, 4933 (2003); 10.1063/1.1559432

Study of light emission from GaN/AIGaN quantum wells under power-dependent excitation J. Appl. Phys. 91, 9622 (2002); 10.1063/1.1476082

Piezoelectric effects on many-body optical gain of zinc-blende and wurtzite GaN/AIGaN quantum-well lasers Appl. Phys. Lett. 75, 1354 (1999); 10.1063/1.124691
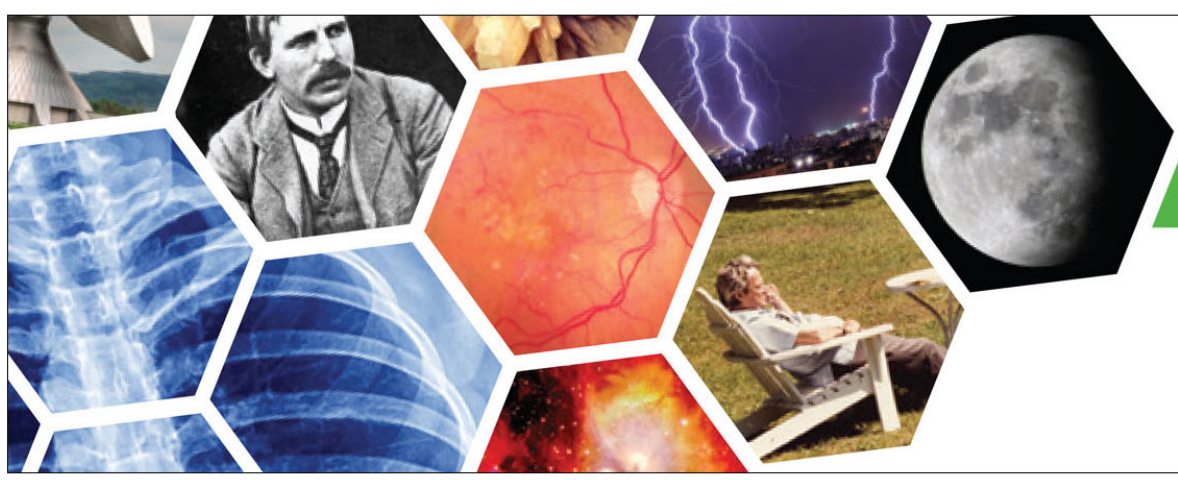

\section{SUBSCRIBE TO physjes today}




\title{
Many-body effects on excitons properties in GaN/AIGaN quantum wells
}

\author{
Giampiero Traetta ${ }^{a)}$ and Roberto Cingolani \\ INFM-Dipartimento di Ingegneria dell'Innovazione, Università di Lecce, 73100 Lecce Italy \\ Aldo Di Carlo, Fabio Della Sala, and Paolo Lugli \\ INFM-Dipartimento di Ingegneria Elettronica, Università di Roma “Tor Vergata," 00133 Roma, Italy
}

(Received 26 July 1999; accepted for publication 26 November 1999)

\begin{abstract}
The many-body effects on excitons properties in GaN/AlGaN quantum wells are theoretically investigated by using a Green's function model and the electron and hole wave functions calculated either in the envelope function approximation or in the frame of a self-consistent tight-binding model. We show that the built-in field induced by the piezoelectric and spontaneous polarization charge causes a reduction of the exciton binding energy and of the absorption coefficient well below the values expected for the quantum well with flat band. At high carrier concentrations, the many-body screening prevails over the screening of the built-in electric field, causing complete exciton bleaching at typical densities of the order of $10^{12} \mathrm{~cm}^{-2}$. (C) 2000 American Institute of Physics. [S0003-6951(00)04004-3]
\end{abstract}

In this letter we present a Green's function model which accounts for the effective potential barrier, the well width, the built-in field, and the screening induced by the injected carriers $^{1-10}$ to study the optical properties of excitons in GaN/AlGaN QWs. The model is an extension of our previous method, ${ }^{11}$ and represents the first comprehensive treatment of the many-body effects in the presence of built-in field and screening. It allows us to calculate the excitonic properties in a continuous way, from the low carrier density, when the built-in field reduces the exciton binding energy through the quantum confined Stark Effect, to the high density limit (the typical operation condition of real devices), when many-body effects induced by a free-carrier plasma screen the Coulomb interaction. The results of this work have a twofold relevance, in that the model is the most comprehensive presented so far, and the properties of the excitons of a strategic material such as GaN/AlGaN QWs are well elucidated. We find that even at very low densities the built-in field reduces the exciton binding energy substantially with respect to the flat band case, with typical values of the order of $10 \mathrm{meV}$ for a $5 \mathrm{~nm} \mathrm{QW}$ with $15 \% \mathrm{Al}$ content in the barrier. Moreover, with increasing carrier density many-body effects further reduce the stability of excitons, which are found to be bleached at densities of the order of $10^{12} \mathrm{~cm}^{-2}$, i.e., well below the typical lasing threshold of these heterostructures.

Our Green function model takes into account the freecarrier screening of the attractive Coulomb potential at finite temperatures and the effective confinement potential in the heterostructure. Furthermore, it provides an exact and analytic expression for the exciton joint density of states which is used to evaluate the integrated area of the exciton band and its oscillator strength. Details on the method can be found in Ref. 11. For the specific case of GaN/AlGaN QWs we have to include the built-in electric field which bends the potential of the QWs and distorts the carrier wave functions. To this aim we have calculated the carrier envelope func-

${ }^{a}$ Electronic mail: giampiero.traetta@unile.it tions, using both the envelope function approximation and a self-consistent tight-binding model.

The total polarization was evaluated as $P_{\text {tot }}=P_{\text {spont }}$ $+P_{\text {piezo }}$, where $P_{\text {piezo }}$ is the piezoelectric charge caused by the lattice mismatch and by the thermal strain, and $P_{\text {spont }}$ is the spontaneous polarization at the GaN/AlGaN interface..$^{7-9}$ For a multiple QW structure (which is the system we consider in the following calculations) the total electric field in the well and in the barrier can be calculated as

$$
F^{w, b}=L^{b, w}\left(P_{\mathrm{tot}}^{b, w}-P_{\mathrm{tot}}^{w, b}\right) /\left[\epsilon_{0}\left(L^{w, b} \epsilon^{b, w}+L^{b, w} \epsilon^{w, b}\right)\right],
$$

where $\epsilon^{b, w}$ is the relative dielectric constant in the barrier $(b)$ and in the well $(w)$ and $L^{w, b}$ is the well (barrier) thickness. The thickness of the barrier is fixed at $5 \mathrm{~nm}$.

Following Ref. 12 for the evaluation of the strain, the built-in field in the wells turns out to vary in the range 1.02.5 MV/cm, depending on the actual well width and $\mathrm{Al}$ content in the barrier (for $x$ varying between 0.15 and 0.3 ). ${ }^{13,14}$

For the envelope function approximation we have solved numerically the Schroedinger equation taking into account the effective potential profile induced by the electric fields in the wells and in the barriers.

However, despite its simplicity, it is well known that the envelope function approximation is not very accurate for narrow wells and cannot be applied to the case of high carrier density if coupling with Poisson equation is not properly accounted for. In particular, the presence of high electric fields, the peculiar band structure of wurtzite semiconductors and the screening of the internal field by the injected charges, which determines a charge redistribution, limit the applicability of the usual envelope function approaches. To overcome all the limitations we have used a self-consistent tightbinding model ${ }^{10,13-15}$ In particular we have performed selfconsistent $s p^{3} d^{5} s^{*}$ tight-binding calculations to determine the electron and hole distribution and the internal electrical field. The electron and hole quasi-Fermi levels are calculated for a given photoinjected charge density. The resulting electron and hole distribution functions ( $n$ and $p$ ), respectively, are used to solve the Poisson equation 


$$
\frac{d}{d z} D=\frac{d}{d z}\left(-\epsilon \frac{d}{d z} V+P_{\text {tot }}\right)=e(p-n)
$$

where $D$ and $V$ are the displacement field and the potential, and $P_{\text {tot }}$ is the total polarization charge discussed earlier. Periodic boundary conditions for the potential $V$ are used to solve Eq. (2). ${ }^{14,15}$

The matrix elements of the potential that takes into account the finite thickness of the quantum well and the freecarrier screening are given by

$$
V_{s}(q)=\left[\frac{V(q)}{\epsilon(q)}\right] F(q),
$$

where $V(q)$ is the unscreened potential, $\epsilon(q)$ is the dielectric constant, and $F(q)$ is the form factor

$$
F(q)=\int d z_{e} \int d z_{h}\left|\chi_{e}\left(z_{e}\right)\right|^{2}\left|\chi_{h}\left(z_{h}\right)\right|^{2} e^{-|q|\left|z_{r}-z_{h}\right|},
$$

which is computed numerically by using the wave functions of the electron and the hole along the $z$-axis $\left[\chi_{e, h}(z)\right]$. The model was tested on II-V and III-V positive-intrinsicnegative structures, ${ }^{16,17}$ giving results for the exciton binding energy and oscillator strength in excellent agreement with the experimental data.

In Fig. 1 we compare the well-width dependence of the exciton binding energy in QWs with flat bands and with the inclusion of the built-in field, for different $\mathrm{Al}$ contents. In these calculations the carrier density is assumed to be $10^{6} \mathrm{~cm}^{-2}$, so that many-body interactions are not important. Our results show that: (a) the exciton binding energy is substantially reduced by the built-in electric field, up to $30 \%$ of the flat band value in the range of well width considered here; (b) the existence of the built-in field causes a change in the shape of the well-width dependence of the exciton binding energy with respect to the flat band case; (c) the exciton binding energies calculated by the envelope function approximation or by the tight-binding model are very close for wide wells, but they are different for narrow wells; and (d) the enhancement of the exciton binding energy observed in QWs with $\mathrm{Al}$ rich barriers in the flat band case is reduced by the electric field. Moreover for wells wider than $3 \mathrm{~nm}$, the binding energies of the $\mathrm{GaN} / \mathrm{Al}_{0.3} \mathrm{Ga}_{0.7} \mathrm{~N}$ QWs are smaller than those of $\mathrm{GaN} / \mathrm{Al}_{0.15} \mathrm{Ga}_{0.85} \mathrm{~N}$ heterostructures, indicating that the effects of the built-in fields are more important than the increase of the band offset. We should point out that for very narrow QWs, the exciton binding energies, obtained in the tight-binding context, are higher than those calculated by the envelope function approach. This effect, already noticed in InGaAs/GaAs systems, ${ }^{14}$ imposes some limitation to the use of the envelope function approach for the calculation of the exciton properties of narrow wells. This is particularly true in nitride based QWs since the polarization fields always confine electrons in a narrow region of the wells (close to the edge of the triangular well shape induced by the polarization fields).

In Fig. 2 we show the well-width dependence of the integrated exciton absorption, calculated by multiplying the square overlap and the integrated joint density of states ${ }^{18,19}$ obtained by our Green's function model, ${ }^{11}$ and normalized to the flat band case one. The normalized exciton absorption of

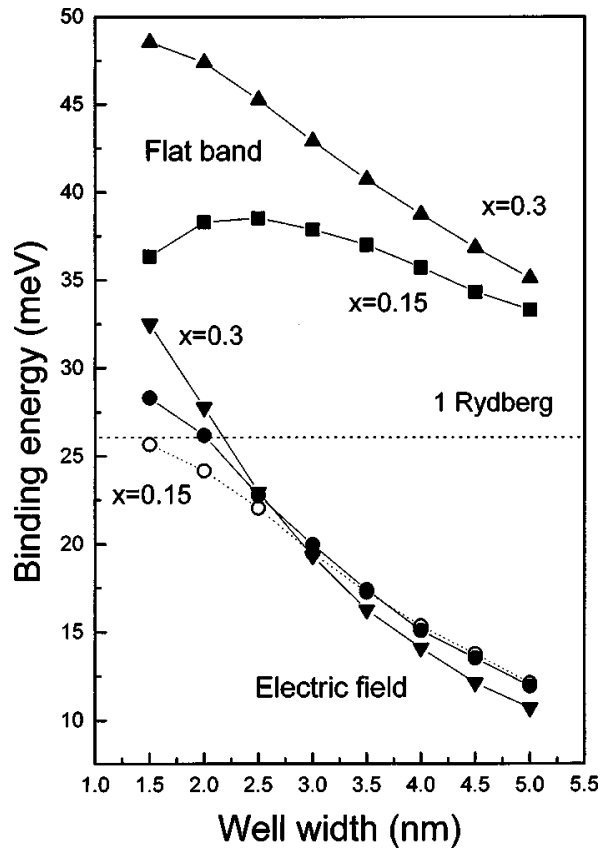

FIG. 1. Well-width dependence of the exciton binding energy for GaN/Al ${ }_{x} \mathrm{GaN}_{1-x} \mathrm{~N}$ QWs with: $x=15 \%$, flat band case $(\boldsymbol{\square}) ; x=30 \%$ flat band case $(\boldsymbol{\Delta}) ; x=15 \%$, with built-in electric field, tight-binding wave functions (O) or envelope function approximation $(\bigcirc) ; x=30 \%$, with built-in electric field and tight-binding wave functions $(\boldsymbol{\nabla})$. The barrier width is fixed to $5 \mathrm{~nm}$.

the $x=0.15$ wells is found to be about 0.25 for $L^{w}=1.5 \mathrm{~nm}$ well and to reduce down to $10^{-3}$ for the $L^{w}=5 \mathrm{~nm}$ well, due to the increased separation of the wave functions occurring in wider wells.

These results clearly show the abrupt reduction of the exciton stability caused by the built-in field in GaN/AlGaN QWs. This might explain why so far a clear evidence of exciton bands is lacking in the photoluminescence excitation or absorption spectra of nitrides QWs, in spite of the strong exciton binding energy of the bulk GaN and of the further enhancement expected for the quantum size effect.

It is, however, important to recall that the polarization charge can be screened by the injection of carriers, ${ }^{10}$ so that under suitable injection conditions one might expect that the screening of the built-in field causes a recovery of the exciton binding energy and oscillator strength. In particular, the injected carriers induce two opposite effects: first, they screen the polarization charge at the interfaces and the internal electric field. This should increase the exciton oscillator strength and binding energy, thus inducing a recovery of the exciton peak. Second, the Coulomb interaction between the electron and the hole is reduced owing to the well-known plasma screening effects. These two effects are obviously competing, and it is not a priori clear which one prevails and determines the excitonic properties of nitride QWs at high carrier densities.

To this aim it becomes very important to include manybody interactions in our calculations, in order to model the exciton properties as a function of the carrier density in the presence of screening and built-in field. The calculations are done in the static limit of the Single Plasmon Pole Approximation. In this limit, the dielectric function in Eq. (3) reads ${ }^{20}$ 


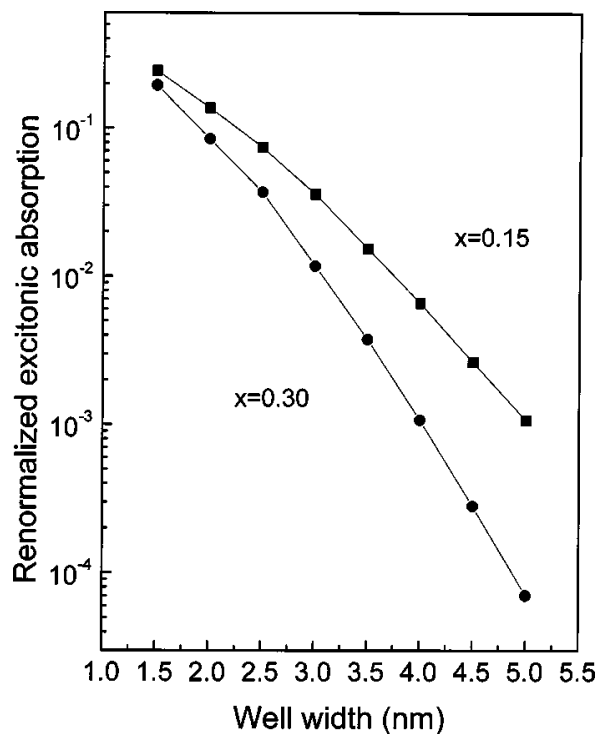

FIG. 2. Well-width dependence of the integrated exciton absorption normalized to the absorption of the flat band case for $\mathrm{GaN} / \mathrm{Al}_{0.15} \mathrm{Ga}_{0.85} \mathrm{~N}(\boldsymbol{\square})$ and $\mathrm{GaN} / \mathrm{Al}_{0.3} \mathrm{Ga}_{0.7} \mathrm{~N}(\bullet)$. The barrier width is fixed to $5 \mathrm{~nm}$.

$$
1 / \epsilon(q)=1-\omega_{\mathrm{pl}}^{2} / \omega_{q}^{2},
$$

where

$\omega_{\mathrm{pl}}^{2}=2 \pi e^{2} n q / \epsilon_{0} \mu$,

$\omega_{q}^{2}=\omega_{\mathrm{pl}}^{2}[1+(q / \kappa)]+q^{2}\left(\hbar^{2} q^{2} / 2 \mu\right)^{2} V(q) F(q) / 2 \pi \hbar^{2} R_{y}$,

( $\mu$ is the reduced mass and $\kappa$ is the inverse screening length). In this way, we can account for both the presence of the electric field induced by piezoelectric and spontaneous polarization, and the many-body effects to describe the exciton properties of GaN/AlGaN QWs.

The density dependent binding energy for two representative well widths $\left(L^{w}=2\right.$ and $\left.L^{w}=4 \mathrm{~nm}\right)$ and $\mathrm{Al}$ contents $(x=0.15$ and $x=0.3)$ is shown in Fig. 3. With increasing the plasma density we see that the exciton binding energy is strongly reduced, suggesting that the many-body bleaching of the exciton occurs at densities for which the screening of the built-in field is still not very effective. Consequently, excitons in nitrides QWs are at not very stable at high density, and specifically they are completely bleached at the typical densities for which lasing occurs, i.e., around $10^{13} \mathrm{~cm}^{-2} .^{3}$ As far as the absorption coefficient is concerned, the increase of the square overlap is compensated by the reduction of the integrated area of the joint density of states, so that the overall effect is again a reduction of the total exciton absorption. Exciton-related lasing is thus very unlikely to occur, unless substantial localization of excitons takes place.

In conclusion, we have given a complete picture of the linear and nonlinear optical properties of excitons in nitrides QWs. We have shown that both the exciton binding energy and oscillator strength are dramatically reduced by the built-in electric field. Moreover, at high carrier concentrations, despite the screening of the built-in field, we find that many-body interactions further reduce the exciton stability.

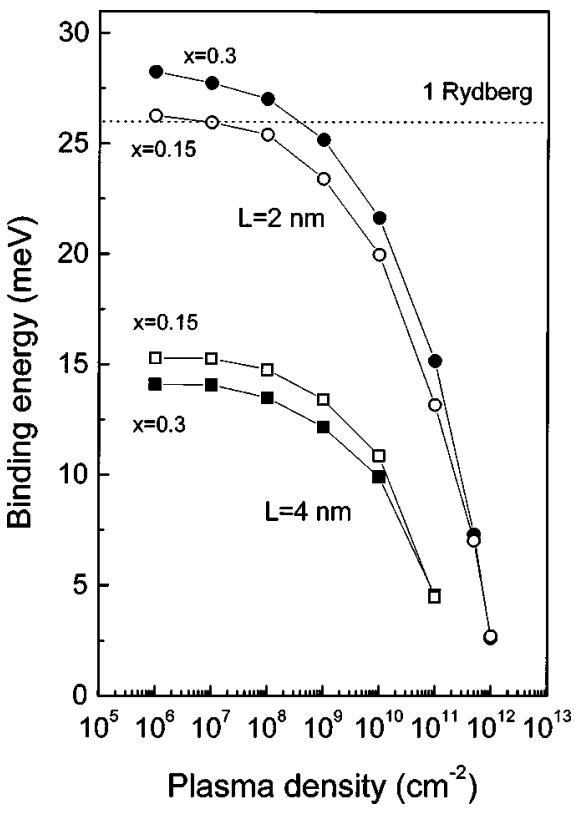

FIG. 3. Carrier density dependence of the exciton binding energy for a $2 \mathrm{~nm}$ wide QW with $x=15 \%(\mathrm{O})$ and $x=30 \%(\bullet)$, and for a $4 \mathrm{~nm}$ wide $\mathrm{QW}$ with $x=15 \%(\square), x=30 \%(\square)$, at $4 \mathrm{~K}$. The barrier width is fixed to $5 \mathrm{~nm}$.

${ }^{1}$ T. Takeuchi, C. Wetzel, S. Yamaguchi, H. Sakai, H. Amano, I. Akasaki, Y. Kaneko, S. Nakagawa, Y. Yamaoka, and N. Yamada, Appl. Phys. Lett. 73, 1691 (1998).

${ }^{2}$ T. Takeuchi, S. Sota, M. Katsuragawa, M. Komori, H. Takeuchi, H. Amano, and I. Akasaki, Jpn. J. Appl. Phys., Part 2 36, L382 (1997).

${ }^{3}$ S. Nakamura and G. Fasol, The Blue Laser Diode (Springer, Berlin, 1997).

${ }^{4}$ S. H. Park and S. L. Chuang, Appl. Phys. Lett. 72, 3103 (1998).

${ }^{5}$ J. S. Im, H. Kollmer, J. Off, A. Sohmer, F. Scholz, and A. Hangleiter, Phys. Rev. B 57, R9435 (1998).

${ }^{6}$ A. Bykhovski, B. Gelmont, and M. Shur, Appl. Phys. Lett. 63, 2243 (1993).

${ }^{7}$ F. Bernardini, V. Fiorentini, and D. Vanderbilt, Phys. Rev. B 56, R10024 (1997).

${ }^{8}$ F. Bernardini, V. Fiorentini, and D. Vanderbilt, Phys. Rev. Lett. 79, 3958 (1997).

${ }^{9}$ F. Bernardini and V. Fiorentini, Phys. Rev. B 57, R9427 (1998).

${ }^{10}$ F. Della Sala, A. Di Carlo, P. Lugli, F. Bernardini, V. Fiorentini, R. Scholz, and J. M. Jancu, Appl. Phys. Lett. 74, 2002 (1999).

${ }^{11}$ G. Traetta, G. Colì, and R. Cingolani, Phys. Rev. B 59, 13196 (1999)

${ }^{12}$ L. Chuang and C. S. Chang, Semicond. Sci. Technol. 12, 252 (1997).

${ }^{13}$ A. Di Carlo, S. Pescetelli, M. Paciotti, P. Lugli, and M. Graf, Solid State Commun. 98, 803 (1996); A. Di Carlo Mater. Res. Soc. Symp. Proc. 491, 389 (1998).

${ }^{14}$ R. Cingolani, A. Botchkarev, H. Tang, H. Morkoç, G. Traetta, G. Coli, M. Lomascolo, A. Di Carlo, F. Della Salla, and P. Lugli, Phys. Rev. B 61, 2711 (2000).

${ }^{15}$ A. Bonfiglio, M. Lomascolo, G. P. Traetta, R. Cingolani, A. Di Carlo, F. Della Sala, P. Lugli, A. Botchkarev, and H. Morkoç, J. Appl. Phys. (in press).

${ }^{16}$ M. Di Dio, M. Lomascolo, A. Passaseo, C. Gerardi, C. Giannini, A. Quirini, L. Tapfer, P. V. Giugno, M. De Vittorio, D. Greco, A. L. Convertino, L. Vasanelli, R. Rinaldi, and R. Cingolani, J. Appl. Phys. 80, 482 (1996).

${ }^{17}$ P. V. Giugno, M. De Vittorio, R. Rinaldi, R. Cingolani, F. Quaranta, L. Vanzetti, L. Sorba, and A. Franciosi, Phys. Rev. B 54, 16934 (1996).

${ }^{18}$ P. Y. Yu and M. Cardona, Fundamentals of Semiconductors (Springer, Berlin, 1996).

${ }^{19}$ J. Sing, Semiconductor Optoelectronics, Physics and Technology (McGraw-Hill, New York, 1995).

${ }^{20}$ G. Colì and R. Cingolani, Solid State Commun. 110, 293 (1999). 